\title{
Electrophysiological Brain Responses of Six-Month-Old Low Risk Premature Infants
}

\author{
Margarita Stolarova \\ University of Konstanz, Konstanz, Germany
}

\author{
Heather Whitney, Sara J. Webb, Raye-Ann deRegnier, \\ Michael K. Georgieff, and Charles A. Nelson \\ Institute of Child Development \\ University of Minnesota
}

\begin{abstract}
This study sought to examine the electrophysiological correlates of visual perceptual priming in a sample of low risk preterm infants. We compared the ERP data of 20 healthy preterm infants at the age of 6 months (corrected age: 4 months) to those of 20 six-month-old and 20 four-month-old full term infants. The comparison groups represented the preterm infants' chronological and corrected ages respectively. The results showed that the brain responses of the preterm infants at the age of 6 months, specifically the topography and the latency of the Nc component, are more similar to those of their corrected age peers than to those of the chronological age controls.
\end{abstract}

In this study, we examined the electrophysiological brain responses of a group of low risk premature infants. Premature delivery interrupts a period of rapid brain growth (Bourgeois, 2001), and confronts an immature system with a set of atypical experiences, more characteristic of full-term (mature) infants. Studying a population of healthy preterm infants gives us an opportunity to examine the relative influence of experience versus maturation on the processes of neural and behavioral development, and gives us information about developmental preprogramming and developmental plasticity of the infant brain (van Hof-van Duin, Heersema, Groenendaal, Baerts, \& Fetter, 1992). We compared a group of low 
risk premature infants to two control groups of healthy full-term infants representing the corrected and chronological ages of the premature group respectively (4- and 6-month olds). ${ }^{1}$ We assumed that the corrected age peers (4-month-old full-term infants) were at a similar level of biological maturation as the premature infants in this study, while the 6-month-old full-term infants had had approximately the same duration of postnatal experience as the premature infants. By comparing the premature group to each of the two full-term control groups, we hoped to determine the relative developmental stage of the preterm infants. The results reported here are part of a larger study on infant memory development in low and high risk premature infants.

In order to study neural functioning, we recorded event-related potentials (ERPs). ERPs have been employed increasingly in the study of infant cognition for the last 20 years and have been used to interpret the underlying processes for different cognitive tasks, predominantly in areas such as attention (Alho, Sainio, Sajaniemi, Reinikainen, \& Naatanen, 1990), speech discrimination, and early word meaning (Molfese, 1990; Novak, Kurtzberg, Kreuzer, \& Vaughan, 1989), and recognition memory (Nelson, 1994). This methodology has also been successfully applied to the study of clinical populations, such as infants with Down Syndrome (Karrer, Karrer, Bloom, Chaney, \& Davis, 1998), and children with autism (Dawson, Carver, Maltzoff, Pangiotides, McParland, \& Webb, 2002). We focus here on the middle latency negative central $(\mathrm{Nc})$ component, a widely distributed component, maximal over fronto-central leads (e.g., de Haan \& Nelson, 1997; for a recent review see Snyder, Webb, \& Nelson, 2002). The Nc has been shown to reflect a basic orienting process, part of the obligatory attention allocation response (Richards, 2002). As such, it is suitable as a measure of developmental changes in very young infants.

In relation to our goals, we hypothesized that by comparing the ERP patterns of the premature infants, specifically the morphology and topography of their $\mathrm{Nc}$, to those of the two control groups, we would be able to better understand the roles of biological maturation and experience in infant brain development. In particular, there are four possible outcomes to this comparison: The brain responses of the premature infants will (a) be more similar to those of their corrected age counterparts (here full-term 4-month olds), and different in terms of morphology and/or topography from the ERPs of the 6-month-old full-term infants, if a cognitive process is more strongly driven by biological maturation, (b) be more similar to those of their chronological age peers, if experience is the main driving force and the immature systems are capable of benefiting from it, (c) fall "in between"

${ }^{1}$ The term chronological age refers to the infants' age since birth. The term corrected (for prematurity) age describes the infants' age since their projected due date for delivery following a full-term gestation. In this study, the premature infants were tested at 4-months corrected age and approximately 6-months chronological age.
4- and 6-month-old control infants, if both experiential and maturational factors are operating at the same time in an interactive pattern, and (d) be different from both 4- and 6-month-old full-term infants, without showing a tendency for an intermediate developmental stage, if some aspect of the infants' brain organization is altered by the experience of prematurity itself. If this is the case, we would expect the ERPs (here specifically the Nc) of the premature infants to differ in morphology or topography from those of both full-term comparison groups.

Previous research has demonstrated that the development of preterm infants' visual evoked potentials (VEPs) is more closely related to their corrected than to their chronological age (Atkinson, Anker, Rae, Weeks, Braddick, Rennie 2002; Roy, Barsoum-Homsy, Orquin, \& Benoit, 1995). The ERP responses, investigated here using a visual priming paradigm with faces (based on Webb, 2001; Webb \& Nelson, 2001, 2002), depend strongly on basic visual orienting. Thus, we hypothesized that the ERPs of the premature infants would resemble those of the 4-month-old full-term controls in terms of amplitude and latency of the Nc, and would differ from the ERP responses of the 6-month-old full-term infants.

\section{METHODS}

\section{Participants}

Preterm group. Preterm infants qualified for this study if they had been born between the 31 st and the 33rd week of gestation (assessed using the best available obstetric evidence and confirmed by physical examination of the newborn), were appropriate for gestational age (AGA) in size and weight at birth, and had Apgar scores $>7$ at 5 min (Apgar \& James, 1962). Premature infants were excluded if they had experienced an intraventricular hemorrhage (IVH), had been mechanically ventilated for more than $24 \mathrm{hr}$, had neurobiological risk scores (Brazy, Eckerman, Oehler, Goldstein, \& O'Rand, 1991; Contractor, Leslie, Bowen, \& Arnold, 1996) higher than three (scores of six or more place an infant at increased risk for developmental impairments), or if the pregnancy had been complicated by diabetes or maternal alcohol or drug abuse. These inclusion and exclusion criteria ensured that the infants participating in this experimental group did not have a history of significant medical complications other than prematurity.

A total of 59 preterm infants were enrolled in this study. Each premature infant was tested within a week of the infants' 4-month corrected birthday, which means, 4 months after the estimated delivery date ( $M=17$ weeks, range 16 to 18 weeks) at approximately six months chronological age ( $M=25$ weeks, range 24 to 26 weeks), see Table 1 . Of the 59 premature infants tested, 39 were excluded due to: (a) over- or underestimation of their gestational age at birth $(n=8)$, (b) medical complications not apparent at the time of enrollment, but revealed by an additional 
TABLE 1

Mean Age at Testing in Weeks and Mean Number of Trials Per Condition Included in the Individual Averages for Each Group

\begin{tabular}{|c|c|c|c|c|c|}
\hline & \multicolumn{2}{|c|}{ Age at Testing } & \multicolumn{3}{|c|}{$\begin{array}{l}\text { Mean Number of Trials } \\
\text { Per Condition }\end{array}$} \\
\hline & $M$ & Range & $M$ & $S D$ & Range \\
\hline 4-month-old controls ${ }^{\mathrm{a}}$ & 17 weeks & $16-18$ & 14.2 & 2.6 & $10-20$ \\
\hline \multicolumn{6}{|l|}{ Premature infants ${ }^{\mathrm{a}}$} \\
\hline corrected age: & 17 weeks & $16-18$ & & & \\
\hline chronological age: & 25 weeks & $24-26$ & 13.2 & 2.7 & $10-20$ \\
\hline 6-month-old controls ${ }^{\mathrm{a}}$ & 25 weeks & $25-26$ & 15.6 & 3.0 & $11-20$ \\
\hline
\end{tabular}

Note. The two control groups represented the corrected (4 months) and the chronological (6 months) ages of the premature infants. No differences between the groups with regard to trial number were found. Infants with fewer than 10 artifact trials were not included in the grand averages computed for each group and condition. The averages for the different conditions included an equal number of trials per channel within the individual subject's data set. ${ }^{\mathrm{a}} n=20$.

review of the infants' medical records by the experimenters $(n=9)$, (c) excessive data artifact generally caused by infant movement or fussiness $(n=22)$. There were no significant differences between the included and excluded premature infants on any of the collected demographic variables or developmental status. The present sample consisted of 20 preterm infants ( 4 males), born between the 31 st and 33rd week of gestation, who met the inclusion criteria and had more than 10 artifact-free trials per channel and condition ( $M=15$, range 10 to 20 trials). The small percentage of included male infants reflects the fact that male infants tend to have more medical complications than female infants (Lauterbach, Raz, \& Sander, 2001), and, thus, fewer boys met our inclusion criteria.

Full term control groups. The 4- and 6-month-old full-term infants were recruited from a community volunteer participant pool. The full-term 4-month olds serve as a comparison group for the preterm infants' corrected for prematurity age, while the full-term 6-month olds serve as a chronological age comparison group. Infants in the full-term groups were born within 10 days of their estimated delivery date, and were tested within a week of their 4- or 6-month birthdays, respectively, with the mean age at testing: 4-month-olds: $M=17$ weeks, range 16 to 18 weeks; 6-month olds $M=25$ weeks, range 25 to 26 weeks (see Table 1). They were included in the final sample only if they had no history of pre- or perinatal medical complications as reported by their parents at test. A total of 138 full-term control infants were tested (69 four- and 69 sixmonth olds). The data for 81 infants were excluded due to: (1) medical complications, most often gestational diabetes $(n=16)$ and (2) excessive data artifact due to movement or fussiness $(n=65)$. Out of a total of 57 full-term infants without any history of medical complications and with more than 10 artifact-free trials per channel and condition (see Table 1 for mean number of trials per group), a subsample of 40 infants ( 20 four- and 20 six-month olds) were matched to the preterm group according to test order and gender ( 7 infants with recognition memory task first, 4 males in each group).

With regard to the demographic background of the infants in the three groups (Table 2), when subjected to an overall ANOVA, no significant differences emerged in terms of socioeconomic status (SES) as measured by household income and maternal education. No group differences were found with regard to non-parental care the infants received, their feeding history, or primary caregiver. The only variable differentiating the preterm infants and the two control groups was the number of twins included $(40 \%$ in the preterm group, $0 \%$ in the two control groups). Multiple birth tends to be a common cause of prematurity in the gestational age population of interest here (Gardner, Goldenberg, Cliver, Tucker, Nelson, \& Copper, 1995). In contrast, full-term twin gestations are rarer and are more often accompanied by pre- and perinatal medical complications than singleton gestations (Buscher, Horstkamp,

TABLE 2

Demographic Characteristics

\begin{tabular}{llccc}
\hline Demographic Variables & & $\begin{array}{c}\text { 4-Month } \\
\text { Olds }(\%)\end{array}$ & $\begin{array}{c}\text { Preterm } \\
\text { Infants }(\%)\end{array}$ & $\begin{array}{c}\text { 6-Month } \\
\text { Olds (\%) }\end{array}$ \\
\hline Household income & $<\$ 25,000$ & 0 & 15.8 & 0 \\
per year & $\$ 25,000-\$ 65,000$ & 52.6 & 36.8 & 40 \\
& $>\$ 65,000$ & 47.4 & 47.4 & 60 \\
Maternal education & High school degree or less & 10.5 & 5.3 & 5 \\
& 2-year college degree & 21.1 & 15.8 & 15 \\
& or some college & & & \\
& 4-year college degree & 36.8 & 47.4 & 45 \\
Care type & Postgraduate studies & 31.6 & 31.6 & 35 \\
& Home & 88.9 & 63.2 & 66.7 \\
Primary caregiver & Day-care & 11.1 & 36.8 & 33.3 \\
& Mother & 78.9 & 73.7 & 58.8 \\
Breastfeeding for & Father & 0 & 10.5 & 5.9 \\
2 months or more & Both & 21.1 & 15.8 & 35.3 \\
& Yes & 83.3 & 78.9 & 94.4 \\
Multiple birth** & No & 16.7 & 21.1 & 5.6 \\
& Singleton & 100 & 60 & 100 \\
& Twin & 0 & 40 & 0
\end{tabular}

Note. The full-term controls were matched with the preterm infants in terms of gender (4 males in each group) and test order ( 7 infants with recognition task first). The only significant difference $\left({ }^{* *}\right)$ was found in regard to the number of twins in each group. 
Wessel, Chen, \& Dudenhausen, 2000), which would have led to exclusion from the present sample. We can assume that the asymmetries in regard to the number of twins included in the 3 groups reflect the selection criteria used.

\section{Stimuli}

The stimuli were full screen color video images of female faces. The visual angle subtended by the stimuli was approximately $12^{\circ}$. When shown to the participants, each image filled a 13 -in. $(33 \mathrm{~cm})$ monitor. The models posed a neutral facial expression in front of a gray background with a gray scarf covering their clothing.

\section{ERP Recording Procedure}

Infants were seated at a distance of approximately $60 \mathrm{~cm}$ from a computer screen in a darkened room. Each testing session consisted of two experiments: a visual priming paradigm (based on Webb, 2001; Webb \& Nelson, 2003), presented in this analysis, and a recognition paradigm (based on de Haan \& Nelson, 1997), not reported here. The test order was counterbalanced across subjects. The recording ended when the maximum number of available trials (102 in the priming and 100 in the recognition paradigm) was reached or when the participants became too tired or too fussy to continue. Infants were presented with a series of pictures of unfamiliar female faces, each displayed for $500 \mathrm{~ms}$, and followed by $1700-2200 \mathrm{~ms}$ gray screen presentation. In the priming experiment, a total of 62 novel images were shown, 20 of these pictures were primed immediately (the primed stimulus followed immediately after the initial presentation), 20 were primed with a delay (the primed stimulus followed after four intervening trials), and 22 were not repeated. When the infant was not attending, the EEG was not recorded, and the stimuli were repeated until the participant looked back at the screen.

\section{Electrophysiological Recording, Data Reduction} and Data Analysis

The electroencephalogram (EEG) was recorded from 14 scalp locations using silver-silver-chlorided (Ag-Ag-Cl) electrodes and Grass EC2 electrode paste. The electrodes were placed over midline $(\mathrm{Fz}, \mathrm{Cz}, \mathrm{Pz}, \mathrm{Oz})$ and lateral $(\mathrm{F} 3, \mathrm{~F} 4, \mathrm{C} 3, \mathrm{C} 4$, $\mathrm{T} 3, \mathrm{~T} 4, \mathrm{~T} 5, \mathrm{~T} 6, \mathrm{P} 3, \mathrm{P} 4)$ scalp positions according to the international 10-20 system (Jasper, 1958). The data were referenced to $\mathrm{Cz}$ during recording and rereferenced off line to linked mastoids. A ground electrode was placed on the forehead, the electrooculogram (EOG) was recorded from bipolar electrodes placed vertically above and below the infant's right eye. Impedances were accepted if they were less than or equal to $10 \mathrm{k} \Omega$.

The EEG and EOG were recorded using a Grass Neurodata Acquisition system with Model 12A5 amplifiers. The EEG gain was set to 20,000, the EOG to 5,000. A bandpass filter of $0.1-30 \mathrm{~Hz}$ was applied and a $60 \mathrm{~Hz}$ notch filter was engaged. The EEG was sampled at $100 \mathrm{~Hz}$. Each trial consisted of $100 \mathrm{~ms}$ baseline recording, $500 \mathrm{~ms}$ stimulus presentation, $1200 \mathrm{~ms}$ post stimulus recording, and a randomized intertrial interval (ITI) ranging from 500 to $1000 \mathrm{~ms}$.

Individual artifact-free trials were combined to form average waveforms for each subject and each condition (novel vs. primed immediately vs. primed with a delay). Artifact rejection procedures were similar to those described in previous reports (e.g., Webb \& Nelson, 2001, 2002). Infants with fewer than 10 artifact free trials per channel and condition were not included in the grand averages computed for each group and stimulus type. The averages for the different conditions included an equal number of trials per channel within the individual subject's data set (Table 1).

Attention was focused on the middle latency negative component Nc (400$770 \mathrm{~ms}$ ). Frontal, central, and parietal electrodes were subject to an omnibus repeated-measures ANOVA with the within-subject factors CONDITION (Novel, Primed immediately, Primed delayed) and LOCATION (Frontal with Fz, F3 and F4, Central with $\mathrm{Cz}, \mathrm{C} 3$ and $\mathrm{C} 4$, Parietal with $\mathrm{Pz}, \mathrm{P} 3$ and P4), as well as the between-subject factor GROUP (premature infants, four-month and six-month fullterm control groups). Follow-up ANOVAs were conducted for significant main effects and interactions $(p<.05)$, the Greenhouse-Geisser method was applied to correct for violation of sphericity, post hoc tests employed the Bonferroni correction for multiple comparisons.

\section{RESULTS}

The analysis of the average $\mathrm{Nc}$ amplitude revealed a significant main effect of LOCATION $(F(2,114)=59.3, p<.01)$. The follow-up pairwise comparisons showed that the mean average amplitude at frontal and central recording sites was significantly more negative than the average amplitude at parietal sites. A LOCATION $\times$ GROUP interaction, $F(4,354)=7.3, p<.01$, was also observed. The follow-up ANOVAs revealed significant location effects for all three groups, preterm infants, $F(2,38)=50.5, p<.001$, 4-month-old control group, $F(2,38)=$ 14.6, $p<.001$, and 6-month-old full term infants, $F(2,38)=5.6, p<.05$. In the 2 less mature groups, the average amplitude at parietal electrodes was significantly less negative than at frontal, $p<.001$, and at central, $p<.001$, leads. In the 6-month-old group, however, there was no significant difference between parietal and central leads, only between parietal and frontal leads, with parietal leads again showing less negativity. This means that while the $\mathrm{Nc}$ demonstrated the expected distribution with maximal amplitude over fronto-central electrodes in all three 
groups, there is a tendency for decreased negativity at frontal and increased negativity at parietal leads across all conditions in the 6-month-old control group compared to both less mature groups.

The analysis of latency to peak of the Nc demonstrated main effects of GROUP, $F(2,57)=6.1, p<.05$, CONDITION, $F(2,114)=3.2, p<.05$, and LOCATION, $F(2,114)=5.5, p<.01$, as well as CONDITION $\times$ GROUP, $F(4,354)=2.9$, $p<.05$, and LOCATION $\times$ GROUP, $F(4,354)=6.1, p<.01$, interactions. In order to clarify the contributions of the fronto-central versus the parietal electrodes to the group differences of interest, two ANOVAs with CONDITION and LEAD as within-subject factors and GROUP as the between-subject variable for a combined fronto-central sensor group (F3, F4, Fz, C3, C4, and Cz) and for the parietal electrodes $(\mathrm{P} 3, \mathrm{P} 4$ and $\mathrm{Pz})$ were conducted. For the fronto-central leads both the preterm group $(M=582.4 \mathrm{~ms})$ and the four-month-old full-term group $(M=584.3$ $\mathrm{ms}$ ) demonstrated significantly longer latencies to peak minimal amplitude than the 6-month-old full-term group $(M=522 \mathrm{~ms})$. There were no differences between the preterm infants and the four-month-old full-term infants. For parietal electrodes, no group differences were found (see Figure 1 and Figure 2).

\section{Mean Latency of the Nc}

at Frontal, Central and Parietal Leads
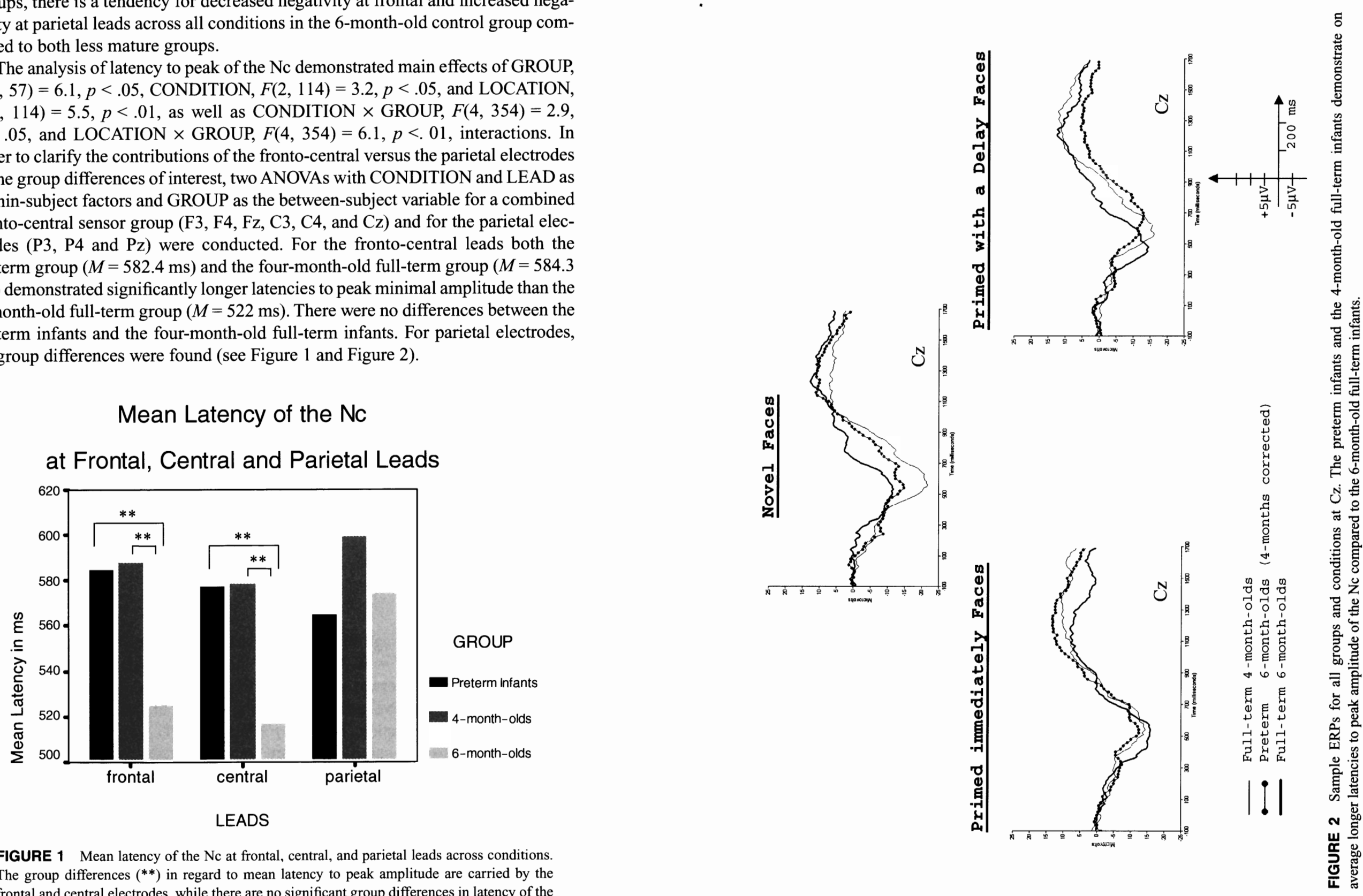

FIGURE 1 Mean latency of the Nc at frontal, central, and parietal leads across conditions. The group differences $\left({ }^{* *}\right)$ in regard to mean latency to peak amplitude are carried by the frontal and central electrodes, while there are no significant group differences in latency of the $\mathrm{Nc}$ at parietal leads. 
Follow-up ANOVAs revealed that the condition effect is carried by the 6-monthold group, main effect of CONDITION, $F(2,38)=12.9, p<.01$. Faces primed with a delay elicited on average significantly longer latencies $(M=637.4 \mathrm{~ms})$ compared to both novel faces $(M=534.2 \mathrm{~ms})$ and primed immediately faces $(M=548.6)$. A CONDITION $\times$ LOCATION interaction, $F(4,76)=11,5, \mathrm{p}<.01$, showed that the latency to peak amplitude at parietal electrodes was very similar across conditions, while for the fronto-central sensor group, the primed with a delay faces elicited a significantly slower response than the other two conditions. No significant main effects or interactions with CONDITION were found for the preterm infants or the 4-month-old full-term group.

In summary, the preterm infants demonstrated longer latencies to peak amplitude at frontal and central leads in comparison with the 6-month-old full-term infants (Figure 1). No significant differences between the preterm and the 4-monthold full-term groups were found. In addition, in the 6-month-old control group, a condition effect of priming was observed: the primed with a delay faces elicited a slower response than the two other conditions. No differences between novel and primed-immediately faces were found. The 2 post-conceptionally less mature groups did not show significant condition effects.

\section{DISCUSSION}

The main goal of this study was to examine the Nc component of the ERP in a group of 6-month-old healthy preterm infants in comparison to those of 2 full-term control groups. The control groups were chosen so that they represented the chronological ( 6 months) and the corrected for prematurity (4 months) ages of the preterm infants. We aimed to determine whether the preterm infants' brain responses were commensurate with their corrected or chronological age. In addition, we hypothesized that the differences between these 3 groups would contribute to our understanding of the relative influence of maturation and experience, with regard to early visual processsing.

The most apparent and stable group differences were found in latency to peak amplitude of the Nc. Across frontal and central leads, the 6-month-old full-term group demonstrated shorter latencies (by an average $60 \mathrm{~ms}$ ) than the 2 less mature groups (Figure 1 and Figure 2). The mean latencies in the preterm group and the 4-month-old full-term group were nearly identical $(M=582.4 \mathrm{~ms}$ and $M=584.3 \mathrm{~ms}$, respectively). Previous research has shown that the latency to peak amplitude for early ERP components decreases throughout childhood (Barnet, 1975; Courchesne, 1978; Eggermont, 1988; Ponton, Eggermont, Kwong, \& Don, 2000). This development toward faster processing has been demonstrated for the auditory, visual, and somatosensory modalities across a variety of experimental designs, and has been interpreted as a general, task-independent, speed of information processing increase, possibly due to the progressing myelination of the corresponding brain areas and their interconnections, or due to an increase in efficiency of the synapses (Eggermont \& Don, 1986).

Our results show that in terms of processing speed, the 6-month-old preterm infants are similar to their corrected age peers and slower than their chronological age controls. It is noteworthy that the latencies obtained in the preterm group were not longer than those in the corrected age control group (4-month-old full-term infants). This finding suggests that moderately premature infants with no additional medical complications do not show evidence of developmental delays in their electrophysiological response to visual stimuli, but appear to develop at the same rate as full-term infants of the same post-conceptional age. Thus, with regard to speed of processing, brain development at this early postnatal stage seems to follow its maturational trajectory, and depend less on experience, regardless of the premature birth.

The analyses of the Nc amplitude also revealed group differences. Specifically, the 6-month-old full-term group showed decreased negativity across frontal electrodes and increased negativity across parietal leads, leading to a reduction of the differences between anterior and posterior recording sites, when compared with each of the 2 less-mature groups. There were no differences between the 4-month full-term controls and the preterm group. The pattern of group differences observed for the amplitude of the $\mathrm{Nc}$ is similar to the group differences for the latency of the $\mathrm{Nc}$ in that the 2 less-mature groups showed very similar responses, while differing significantly from the 6-month-old full-term controls.

In electrophysiological studies of adult cognition, it has been hypothesized that a decrease in amplitude reflects a reorganization towards more automated and efficient processing (Desimone, 1998). Greater negativity of the $\mathrm{Nc}$ at fronto-central leads in infants has previously been interpreted as a sign of greater allocation of attention (Courchesne, Ganz, \& Norcia, 1981). Recently, Richards (2002) provided additional evidence for an increase of the Nc amplitude during attention in infants at ages $4.5,6$, and 7.5 months. Karrer et al. (1998) have shown that 6-month-old infants with Down Syndrome demonstrate larger Nc areas and greater peak amplitudes compared to 6-month-old typically developing infants in an $80 / 20 \%$ oddball paradigm with female faces as stimuli. The authors interpreted this as a sign of slower and less efficient stimulus encoding in the Down Syndrome group. It is likely that the patterns described by Karrer et al., reflect a developmental delay, while the ERPs reported here, for the 2 less mature groups, represent an earlier stage of development compared with the 6-month-old full-term infants. Thus, the change in amplitude of the Nc likely reflects a developmental progression towards greater efficiency.

The electrophysiological methodology used here has proved suitable for tracking developmental changes across the relatively short time span of 2 months. It 
has also helped evaluate indirectly the relative stage of brain development in a clinical infant population. The results of this study indicate that at the chronological age of 6 months, the brain responses of low risk premature infants develop according to their post-conceptional age. At an early postnatal developmental stage this phenomenon seems to be task-independent, and probably reflects general maturationally driven developmental processes, such as increasing myelination and connectivity of the infant brain. It can be expected that, with age, the interaction of maturational and experiential factors will become increasingly important (Katz \& Shatz, 1996; Singer, 1986; Whitney, 2003).

\section{ACKNOWLEDGMENTS}

Research and manuscript preparation were made possible in part by grants from the German National Merit Foundation to Margarita Stolarova, from the Graduate School of the University of Minnesota to Heather Whitney, and from the NIH (NS34458) and the John D. and Catherine T. MacArthur Foundation (through their support of a research network on early experience and brain development) to Charles A. Nelson.

We are particularly indebted to the families of our participants for their commitment to this study, and to the NICU staff at the participating hospitals for their help with screening and recruitment of participants. We would also like to thank the Developmental Cognitive Neuroscience Lab at the University of Minnesota for the support provided during data collection and helpful comments on our work.

\section{REFERENCES}

Alho, K., Sainio, K., Sajaniemi, N., Reinikainen, K., \& Naatanen, R. (1990). Event-related brain potential of human newborns to pitch change of an acoustic stimulus. Electroencephalography and Clinical Neurophysiology, 77, 151-155.

Apgar, V., \& James, L. S. (1962). Further observations on the newborn scoring system. American Journal of Deseases of Children, 104, 419-428.

Atkinson, J., Anker, S., Rae, S., Weeks, F., Braddick, O., \& Rennie, J. (2002). Cortical visual evoked potentials in very low birthweight premature infants. Archives of Disease in Childhood/Fetal and Neonatal Edition, 86, F28-31.

Barnet, A. B. (1975). Auditory evoked potentials during sleep in normal children from ten days to three years of age. Electroencephalograpy and Clinical Neurophysiology, 39, 29-41.

Bourgeois, J. P. (2001). Synaptogenesis in the neocortex of the newborn: The ultimate frontier for individuation? In C. A. Nelson \& M. Luciana (Eds.), Handbook of developmental cognitve neuroscience (pp. 23-33). Cambridge, MA: A Bradford Book, The MIT Press.

Brazy, J. E., Eckerman, C. O., Oehler, J. M., Goldstein, R. F., \& O’Rand, A. M. (1991). Nursery Neurobiologic Risk Score: important factor in predicting outcome in very low birth weight infants. Journal of Pediatrics, 118, 783-792.
Buscher, U., Horstkamp, B., Wessel, J., Chen, F. C., \& Dudenhausen, J. W. (2000). Frequency and significance of preterm delivery in twin pregnancies. International Journal of Gynecology and Obstetrics, 69, 1-7.

Contractor, C. P., Leslie, G. I., Bowen, J. R., \& Arnold, J. D. (1996). The Neonatal Neurobiologic Risk Score: does it predict outcome in very premature infants? Indian Pediatrics, 33, 95-101.

Courchesne, E. (1978). Neurophysiological correlates of cognitive development: changes in longlatency event-related potentials from childhood to adulthood. Electroencephalograpy and Clinical Neurophysiologyl, 45, 468-482.

Courchesne, E., Ganz, L., \& Norcia, A. M. (1981). Event-related brain potentials to human faces in infants. Child Development, 52, 804-811.

Dawson, G., Carver, L., Maltzoff, A., Pangiotides, H., McParland, J., \& Webb, S. (2002, May-June). Neural correlates of face and object recognition in young children with autism spectrum disorder, developmental delay and typical development. Child Development, 73(3), 700-717.

de Haan, M., \& Nelson, C. A. (1997). Recognition of the mother's face by six-month-old infants: a neurobehavioral study. Child Development, 68, 187-210.

Desimone, R. (1998). Visual attention mediated by biased competition in extrastriate visual cortex. Philosophical Transactions of the Royal Society of London/Series B Biological Sciences, 353, $1245-1255$.

Eggermont, J. J. (1988). On the rate of maturation of sensory evoked potentials. Electroencephalograpy and Clinical Neurophysiology, 70, 293-305.

Eggermont, J. J., \& Don, M. (1986). Mechanisms of central conduction time prolongation in brainstem auditory evoked potentials. Archives of Neurology, 43, 116-120.

Gardner, M. O., Goldenberg, R. L., Cliver, S. P., Tucker, J. M., Nelson, K. G., \& Copper, R. L. (1995). The origin and outcome of preterm twin pregnancies. Obstetrics and Gynecology, 85, 553-557.

Jasper, H. H. (1958). The ten-twenty electrode system of the International Federation. Electroencephalograpy and Clinical Neurophysiology, 10, 371-375.

Karrer, J. H., Karrer, R., Bloom, D., Chaney, L., \& Davis, R. (1998). Event-related brain potentials during an extended visual recognition memory task depict delayed development of cerebral inhibitory processes among 6-month-old infants with Down syndrome. International Journal of Psychophysiology, 29, 167-200.

Katz, L. C., \& Shatz, C. J. (1996). Synaptic activity and the construction of cortical circuits. Science, $274,1133-1138$

Lauterbach, M. D., Raz, S., \& Sander, C. J. (2001). Neonatal hypoxic risk in preterm birth infants: the influence of sex and severity of respiratory distress on cognitive recovery. Neuropsychology, 15 , $411-420$.

Molfese, D. L. (1990). Auditory evoked responses recorded from 16-month-old human infants to words they did and did not know. Brain and Language, 38, 345-363.

Nelson, C. A. (1994). Neural correlates of recognition memory in the first postnatal year. In G. Dawson \& K. W. Fischer (Eds.), Human behavior and the developing brain (pp. 269-313). New York: The Guilford Press.

Novak, G. P., Kurtzberg, D., Kreuzer, J. A., \& Vaughan, H. G., Jr. (1989). Cortical responses to speech sounds and their formants in normal infants: maturational sequence and spatiotemporal analysis. Electroencephalograpy and Clinical Neurophysiology, 73, 295-305.

Ponton, C. W., Eggermont, J. J., Kwong, B., \& Don, M. (2000). Maturation of human central auditory system activity: evidence from multi-channel evoked potentials. Clinical Neurophysiology, 111, 220-236.

Richards, J. E. (in press). Attention affects the recognition of briefly presented visual stimuli in Infants: an ERP study. Developmental Science.

Roy, M. S., Barsoum-Homsy, M., Orquin, J., \& Benoit, J. (1995). Maturation of binocular pattern visual evoked potentials in normal full-term and preterm infants from 1 to 6 months of age. Pediatric Research, 37, 140-144. 
Singer, W. (1986). The brain as a self-organizing system. European Archives of Psychiatry, 236, 4-9. Snyder, K., Webb, S., \& Nelson, C. A. (2002). Theoretical and methodological implications of variability in infant brain response during a recognition memory paradigm. Infant Behavior and Development, 25, 466-494.

van Hof-van Duin, J., Heersema, D. J., Groenendaal, F., Baerts, W., \& Fetter, W. P. (1992). Visual field and grating acuity development in low-risk preterm infants during the first $21 / 2$ years after term. Behavioural Brain Research, 49, 115-122.

Webb, S. J. (2001). Perceptual priming in infancy: Evidence from event-related potentials. (Doctoral dissertation, University of Minnesota, 2001). Dissertation Abstracts International, 62 (3-B), 1623.

Webb, S. J., \& Nelson, C. A. (2001). Perceptual priming for upright and inverted faces in infants and adults. Journal of Experimental Child Psychology, 79, 1-22.

Webb, S. J., \& Nelson, C. A. (2003). Facial priming during the first year of life: ERP evidence of a developmental shift. Manuscript submitted for publication.

Whitney, H. (2002). Event-related potentials in low risk preterm infants: A comparison with full-term infants of the same post-conceptual and postnatal age. Manuscript in preparation. 